\title{
The Impact of the 2007 ATS/IDSA Diagnostic Criteria for Nontuberculous Mycobacterial Disease on the Diagnosis of Nontuberculous Mycobacterial Lung Disease
}

\author{
Dong-Ryeol Chae $^{a} \quad$ Yu-Il Kim ${ }^{a}$ Seung-Jung Kee ${ }^{b}$ Yoon-Hee Kim ${ }^{a}$ \\ Su-Young Chi ${ }^{a}$ Hee-Jung Ban ${ }^{a}$ Yong-Soo Kwon ${ }^{a}$ In-Jae Oh ${ }^{a} K_{\text {Kyu-Sik Kim }}{ }^{a}$ \\ Soo-Ock Kim ${ }^{c}$ Young-Chul Kim ${ }^{a}$ Sung-Chul Limª \\ Departments of a Internal Medicine and ${ }^{b}$ Laboratory Medicine, Chonnam National University and Hospital, \\ Gwangju, and 'Department of Internal Medicine, Seonam University College of Medicine, Namwon, Korea
}

\section{Key Words}

ATS/IDSA guidelines $\cdot$ Diagonosis rate $\cdot$ Nontuberculous mycobacterial lung disease

\begin{abstract}
Background: In 2007, the American Thoracic Society (ATS) and Infectious Disease Society of America (IDSA) published new diagnostic guidelines for nontuberculous mycobacterial (NTM) disease. Bacteriological criteria have become simpler compared to the 1997 ATS diagnostic criteria. Objective: For assessing the impact of the 2007 ATS/IDSA diagnostic criteria, we compared the diagnosis rate and time to diagnosis of NTM lung disease using the 1997 and 2007 ATS guidelines. Methods: Sixty-four patients who had excreted Mycobacterium intracellulare, M. avium, M. abscessus or M. kansasii at least one time in their respiratory specimens at Chonnam National University Hospital were reviewed. The 1997 ATS and 2007 ATS/IDSA guidelines were applied to these patients. Results: Thirty-seven of 64 patients (57.8\%) were diagnosed with NTM lung disease by the 1997 ATS criteria. When the 2007 ATS/IDSA criteria were applied, 6 patients were newly diagnosed with NTM lung disease. The diagnosis rate significantly increased from 57.8 to $67.2 \%(p<0.001)$. The
\end{abstract}

time to diagnosis in the 1997 ATS and 2007 ATS/IDSA guidelines was $46.4 \pm 53.0$ and $36.2 \pm 38.5$ days, respectively $(p=0.002)$. Conclusion: These data suggest that we can shorten the time to diagnose NTM lung disease and diagnose more simply by using the 2007 ATS/IDSA guidelines. Further study will be needed to assess that these changes affect the management of NTM disease.

Copyright $\odot 2010$ S. Karger AG, Base

\section{Introduction}

Mycobacteria other than Mycobacterium tuberculosis complex and $M$. leprae are referred to as nontuberculous mycobacteria (NTM) [1]. NTM are environmental organisms found primarily in water, both natural and tap water, but are also found in soil, dust, animals and food [2, 3]. The increase in the prevalence of NTM infections in non-HIV patients has been observed over time in many countries, although there are regional differences in species distribution and clinical relevance [4-6]. In South Korea, the number of patients with clinical disease caused by NTM also continues to increase [7].

\section{KARGER}

Fax +4161306 1234

E-Mail karger@karger.ch

www.karger.com (c) 2010 S. Karger AG, Basel

$0025-7931 / 11 / 0822-0124 \$ 38.00 / 0$

Accessible online at:

www.karger.com/res
Yu-Il Kim, MD, Department of Internal Medicine

Division of Pulmonology and Critical Care Medicine

Chonnam National University and Hospital, 8, Hak-dong, Dong-gu

Gwangju 501-757 (South Korea)

Tel. +82 62220 6296, Fax +82 62225 8578, E-Mail kyionly@ chonnam.ac.kr 
The diagnosis of NTM lung disease is more complex than that of pulmonary tuberculosis. The complexity of the diagnosis is due to the indolent nature of the disease, nonspecific symptoms and mainly the fact that the single identification of NTM on respiratory specimens is not sufficient for diagnosis. According to the American Thoracic Society (ATS) guideline for NTM disease published in 1997, at least three positive sputum cultures are needed for diagnosis of NTM lung disease [1]. However, ATS and the Infectious Disease Society of America (IDSA) have published new diagnostic guidelines for NTM disease that have stricter radiological criteria in 2007 [2]. The new 2007 diagnostic guidelines have become simpler and more lenient on bacteriological criteria: two positive sputum cultures or a single NTM culture from bronchial washing fluid are now enough to diagnose NTM lung disease, especially for the M. avium complex (MAC; $M$. intracellulare and M. avium), M. kansasii and M. abscessus. We hypothesize that more patients with NTM infection will be diagnosed with NTM disease and have a shorter time to be diagnosed. However, there is only one brief report about the impact of the 2007 guidelines as compared with the 1997 guidelines [8]. There is no data comparing the two guidelines for the three most common NTM: MAC, M. kansasii and M. abscessus.

To evaluate the impact of the 2007 ATS/IDSA guideline on the diagnosis of NTM lung disease, we compared the diagnosis rate and time to diagnosis of NTM lung disease using the 1997 versus 2007 guidelines by reviewing medical records of all patients in whom NTM was isolated from respiratory specimens.

\section{Materials and Methods}

\section{Subjects and Design of the Study}

The medical records of patients who had excreted NTM at least once and had the identified species of NTM in respiratory specimens including sputum and bronchial washing fluid at Chonnam National University Hospital, South Korea, during the 2-year period from January 2006 to December 2007 were reviewed. Then, the reviewed data were stored in a protected and anonymized database for each subject to maintain patient confidentiality and the study was approved by our local IRB. The diagnosis of NTM lung disease was made by the diagnostic criteria published by the ATS in 1997 [1]. The guidelines were applied to the patients who had excreted MAC, M. kansasii or M. abscessus as the identified species of NTM. During the same period, the 2007 ATS/IDSA guidelines [2] for NTM lung disease were applied to these patients. Then we compared the time to diagnosis, which describes the time from the first visit day with respiratory symptoms related to NTM lung disease to the day when the diagnosis was established, according to each diagnostic guideline. We also observed the development of the newly diagnosed patients by the 2007 ATS/IDSA guidelines.

NTM isolates recovered from nonrespiratory specimens, including urine, stool and joint fluid, were excluded from this study.

\section{Acid Fast Bacillus Smears and Cultures}

All respiratory specimens were digested and decontaminated according to the guidelines provided by the ATS [9]. Specimens were processed by the $\mathrm{N}$-acetyl-L-cystein $\mathrm{NaOH}$ method.

Smears were stained with auramine and examined with a fluorescence microscope. The results of fluorochrome smears were reported semiquantitatively. A positive smear was graded from $1+$ (1-9 organisms per 10 high-power fields) to $4+(>90$ organisms per high-power field) [9].

Processed specimens were inoculated into the broth system, BACTEC MGIT 960 system (Becton Dickinson, Sparks, Md., USA) [10], to detect mycobacterial growth. Any sample which was identified as positive was removed from the instrument, and a smear was prepared and examined for acid fast bacillus (AFB).

\section{Identification of NTM Species}

NTM species identification was performed using a multiplex polymerase chain reaction (PCR) - restriction fragment length polymorphism method $[11,12]$. After the extraction of genomic DNA from an isolate, we performed multiplex PCR using primer targeting the $16 \mathrm{~S}$ rRNA gene and the MPB70 gene. If only the $16 \mathrm{~S}$ rRNA gene was amplified, the isolate was confirmed to be NTM. In the next step, we performed multiplex PCR using primers of $M$. avium, $M$. intracellulare, $M$. kansasii, $M$. fortuitum, $M$. abscessus/ chelonae, M. terrae, and M. gordonae. If M. abscessus/chelonae were identified in this multiplex PCR, the PCR products were processed for the restriction fragment length polymorphism method using Hae III restriction enzyme (Takara Shuzo, Shiga, Japan). According to the sizes of the restricted fragments, we differentiated M. abscessus from M. chelonae. Among the identified species, we included 4 species, $M$. intracellulare, $M$. avium, $M$. abscessus and M. kansasii, to diagnose and analyze the data using the ATS/ IDSA guidelines as the diagnostic criteria fit best with those 4 species [2].

\section{Statistical Analysis}

The differences of time to diagnosis and change of rate of diagnosis between the two groups were compared by the paired $t$ test and $\chi^{2}$ test, respectively. We used the SPSS software package version 17.0 and a $p$ value of $<0.05$ was considered statistically significant.

\section{Results}

Between 2006 and 2007, 68 patients were found to have positive NTM culture and identified species of NTM from their respiratory specimen at least once. Four patients among them, 2 cases of $M$. chelonae and 2 cases of M. gordonae, were excluded and 64 patients were included in the analysis of this study (table 1). Thirty-seven of the 64 patients (57.8\%) were diagnosed with NTM lung 
disease by the 1997 ATS diagnostic guidelines. When the 2007 ATS/IDSA guidelines were applied to the 64 patients, 6 patients $(9.4 \%)$ were newly diagnosed with NTM lung disease (table 1). The rate of diagnosis was significantly increased from 57.8 to $67.2 \%$ when the 2007 ATS/ IDSA guideline was used ( $\mathrm{p}<0.001)$. The 4 most frequently isolated species were, in decreasing order, $M$. intracellulare, $M$. avium, M. abscessus and M. kansasii. And 2 cases had mixed infection with M. abscessus and M. intracellulare.

The clinical characteristics of the 37 patients with NTM lung disease by the 1997 guidelines are shown in table 2 . The frequent comorbidities were old pulmonary tuberculosis (previous inactive tuberculosis), chronic obstructive pulmonary disease, diabetes mellitus and AIDS, in decreasing order. Most of them (78.3\%) had cough or sputum.

The clinical characteristics of the newly diagnosed patients with NTM lung disease by the 2007 ATS/IDSA guidelines are shown in table 3 . Two of six patients had two positive sputum cultures, while AFB smears were negative in these 2 patients. These patients are compatible to the 2007 but not the 1997 guidelines to diagnose NTM lung disease. Four other patients had only single positive culture results of bronchial washing fluid. However, AFB smear results were also negative so that these patients could not be diagnosed with NTM lung disease using the 1997 diagnostic criteria. Among the 6 patients, 3 did not show more positive NTM culture on the follow-up cultures. These 3 patients underwent chest computed tomography follow-up after a year of diagnosis without treatment of NTM, and there was no evidence of disease progression. The other 3 patients were lost to follow-up.

The time to diagnosis in the 1997 ATS and 2007 ATS/ IDSA guidelines was $46.4 \pm 53.0$ and $36.2 \pm 38.5$ days, respectively. When we used the 2007 ATS/IDSA guidelines, the diagnosis of NTM lung disease was established approximately 10 days earlier. There is a statistically significant difference in the time to diagnosis $(\mathrm{p}=0.002)$.

\section{Discussion}

NTM are being increasingly recognized as an important cause of chronic lung infection in many countries. However, there are geographical differences in the epidemiological features of NTM disease [2]. Recently, van Ingen et al. [4] reported that in NTM patients in the Netherlands, who were mostly aged men with chronic obstructive pulmonary disease, $M$. avium was the most
Table 1. Diagnostic rates of NTM pulmonary disease in the 1997 and 2007 ATS guidelines by species

\begin{tabular}{lrcc}
\hline Organism & $\mathrm{n}$ & 1997 ATS + & 2007 ATS + \\
\hline MAC & & & \\
$\quad$ M. intracellulare & 42 & $21(50.0)$ & $26(61.9)$ \\
$\quad$ M. avium & 12 & $8(66.7)$ & $9(75.0)$ \\
M. abscessus & 4 & $3(75.0)$ & $3(75.0)$ \\
M. kansasii & 4 & $3(75.0)$ & $3(75.0)$ \\
M. abscessus, MAC & 2 & $2(100.0)$ & $2(100.0)$ \\
\hline Total & 64 & $37(57.8)^{2}$ & $43(67.2)^{2}$ \\
\hline
\end{tabular}

Figures in parentheses are percentages.

${ }^{1}$ Mixed infection with M. abscessus and MAC.

2 The difference of NTM lung disease diagnosis rates by the 1997 vs. 2007 guidelines was significant ( $<<0.001)$.

Table 2. Clinical characteristics of patients with NTM lung disease by the 1997 ATS guidelines

\begin{tabular}{ll}
\hline Characteristics & $\begin{array}{l}\text { Patients with NTM } \\
\text { lung disease }(\mathrm{n}=37)\end{array}$ \\
\hline $\begin{array}{l}\text { Mean age, years } \\
\text { Sex }\end{array}$ & 61.8 \\
$\quad$ Male & $16(43.2 \%)$ \\
Female & $21(56.7 \%)$ \\
Smoker & $16(43.2 \%)$ \\
Comorbidity & \\
Old pulmonary tuberculosis & $21(56.7 \%)$ \\
COPD & $12(32.4 \%)$ \\
Bronchiectasis & $10(27.0 \%)$ \\
Diabetes mellitus & $6(16.2 \%)$ \\
AIDS & $1(2.7 \%)$ \\
Symptom & \\
Cough/sputum & $29(78.3 \%)$ \\
Hemoptysis & $9(24.3 \%)$ \\
Dyspnea & $7(18.9 \%)$ \\
Chest discomfort & $4(10.8 \%)$ \\
\hline
\end{tabular}

common organism. In the United Kingdom, M. kansasii is the most common pathogen, especially in England and Wales [13]. In recent reports from the USA, $M$. intracellulare is the most common respiratory pathogen in nonHIV patients, and the nodular/bronchiectatic MAC disease has been reported predominantly in middle-aged to elderly nonsmoking women without underlying predisposing conditions $[2,14,15]$. In our study, older nonsmoking women were predominantly diagnosed as having NTM lung disease and $M$. intracellulare was the most 
Table 3. Clinical and microbiological summary of patients who were newly diagnosed by the 2007 ATS/IDSA guidelines

\begin{tabular}{|c|c|c|c|c|c|}
\hline Age/sex & Symptom & Specimens & NTM identification & Initial CT findings & $\begin{array}{l}\text { Follow-up } \\
\text { CT findings }\end{array}$ \\
\hline $64 / \mathrm{M}$ & cough, sputum, hemoptysis & sputum (2) & M. intracellulare & nodular bronchiectasis & lost \\
\hline $62 / \mathrm{F}$ & cough, sputum & washing (1) & M. intracellulare & cavity & lost \\
\hline $62 / \mathrm{F}$ & cough, sputum, hemoptysis & sputum (1), washing (1) & M. intracellulare & bronchiectasis & no change \\
\hline
\end{tabular}

common pathogen (table 2). This was the case even though many patients had underlying comorbidities such as old pulmonary tuberculosis because of a relatively high prevalence of tuberculosis in Korea. These features in our study are similar to other reports from Korea [7].

The diagnosis of NTM lung disease is often difficult due to the insidious and indolent nature of this disease. Moreover, the one-time isolation of NTM from sputum or bronchoalveolar lavage fluid is often considered as contaminated or colonized with NTM, especially in immunocompetent patients with bronchiectasis [16]. Generally, NTM lung disease is slowly progressive. There is usually sufficient time to collect adequate respiratory specimens necessary for making a diagnosis. However, because some patients with nodular bronchiectatic NTM lung disease produce minimal amount of sputum, it is difficult to diagnose using the 1997 ATS guidelines [17]. Even with bronchoscopy, the diagnosis may be elusive [17, 18]. Also, there have been some debates about the concept of airway colonization by NTM in nodular bronchiectatic disease. The new ATS/IDSA diagnostic guideline was published in 2007 based on these prior studies. The most significant difference between the two guidelines is the change in microbiological criteria. In the new ATS/ IDSA guidelines, two positive sputum cultures or a single positive culture from bronchial washing fluid are enough to establish the diagnosis of NTM lung disease. Previous guidelines included the recommendations based on quantification of smears and cultures. In the new ATS/ IDSA guideline, this was removed because quantitative smear and culture results were not available in many laboratories, especially those using only liquid culture media.

In a brief report [8], the authors used the new 2007 ATS/IDSA guidelines and assessed the impact on the diagnosis of NTM lung disease by retrospective means. Approximately $7.7 \%$ more pulmonary patients met the new
2007 ATS/IDSA guideline than the 1997 guideline, although MAC species were not included, this differed by NTM species, and the 2007 guideline contained more specific radiological criteria. The authors commented that as more patients are likely to meet the new ATS/ IDSA guideline, more patients might receive antimycobacterial treatment. But the change of rate of antimycobacterial treatment was not described in the report. The authors of the new ATS/IDSA diagnostic guideline also agreed that more patients will likely be diagnosed with NTM lung disease as a result of the new ATS/IDSA diagnostic guideline [19]. These reports could be supportive of our study that included MAC species. We observed similar findings from our patients. The increase in newly diagnosed patients was 6 patients, $9.4 \%$ of total patients $(\mathrm{p}<0.001)$ in our study, which was slightly higher than in the previous report [8]. This difference may be due to different species that were included in the analysis and different prevalence of NTM lung disease according to the country. Additionally, our study showed that the time to diagnosis was shortened by using the new 2007 guidelines $(\mathrm{p}=0.002)$. It is obvious that the lesser number of samples needed for diagnosis results in shorter time to diagnosis in the new guidelines. We do not need to take additional sputum or other tests after two positive culture results according to the 2007 criteria. Therefore, we could expect to lower the number of tests and decrease the time to diagnose NTM disease.

Because the diagnosis is established more easily and quickly, it is possible that the new guidelines will influence the start of treatment. However, the fact that diagnosis is not always followed by treatment because of the indolent nature of this disease is well known. Three newly diagnosed patients by the 2007 guidelines have not shown the prominent clinical deterioration or the changes of computed tomography lesions in our study until now, although these patients were not treated with anti- 
mycobacterial drugs. The necessity of therapy is unknown, as these 3 patients would not have benefitted from therapy. If there had been any patients with disease progression, it would have been supportive of the predictive value of the 2007 diagnostic criteria and therapy would have begun. Overall, these patients are a good demonstration of the difficulty of managing patients with NTM lung disease, even after they have been diagnosed. The treatment decision is complicated and it could be a separate consideration from the diagnosis of NTM lung disease. Further study will be needed to determine the impact on treatment. We preliminarily compared the time to treatment between the patients who were diagnosed in this study and the patients who were diagnosed after 2007. The time to treatment describes the time from the first visit day with respiratory symptoms related to NTM lung disease to the day when first antimycobacterial drugs are administered to the patient. Although there was no statistical significant difference $(\mathrm{p}=0.571)$, the time to treatment was shortened by approximately 10 days in the patients who were diagnosed after 2007 (unpubl. data). This observation suggests that a more lenient diagnostic guideline may not only influence time to diagnosis but also time to treatment in the future. This is, however, limited because this study is only retrospective and not a contemporary observation. There are other recent reports suggesting that the earlier diagnosis or therapy is done, the better the outcomes will be [20,21]. In the future, further study is needed to evaluate the two follow- ing questions. Do we treat earlier and more patients with the new criteria? Do we improve the prognosis of these patients?

This study has several limitations. First, this is a retrospective study that was conducted at a single center with a relatively small population size. Prospective and multicenter studies including the impact on treatment will be needed. Second, we did not include the extrapulmonary NTM and did not include species other than the 3 mentioned: MAC, M. abscessus, M. kansasii. However, our study could be meaningful because the three most common species were included and pulmonary disease is one of the most common forms of NTM disease.

In conclusion, more patients were diagnosed with NTM lung disease and the time to diagnosis was shortened by approximately 10 days when the 2007 ATS/IDSA guideline was used. These results suggest that we can shorten the time to diagnose NTM lung disease and that we can diagnose more simply by using the 2007 ATS/ IDSA guidelines. However, further evaluation is needed about the impact of the 2007 ATS/IDSA guideline on management and prognosis of NTM lung disease.

\section{Acknowledgements}

The authors thank Hee-Young Shin in the Clinical Trial Center for statistical support as well as Rebecca Oberley and Jon Danley for review of the manuscript and suggestions.

\section{References}

1 Diagnosis and treatment of disease caused by nontuberculous mycobacteria. This official statement of the American Thoracic Society was approved by the Board of Directors, March 1997. Medical Section of the American Lung Association. Am J Respir Crit Care Med 1997;156:S1-S25.

$\checkmark 2$ Griffith DE, Aksamit T, Brown-Elliott BA, Catanzaro A, Daley C, Gordin F, Holland SM, Horsburgh R, Huitt G, Iademarco MF, Iseman $\mathrm{M}$, Olivier $\mathrm{K}$, Ruoss $\mathrm{S}$, von Reyn $\mathrm{CF}$ Wallace RJ Jr, Winthrop K: An official ATS/ IDSA statement: diagnosis, treatment, and prevention of nontuberculous mycobacterial diseases. Am J Respir Crit Care Med 2007; 175:367-416.

3 Ichiyama S, Shimokata K, Tsukamura M: The isolation of Mycobacterium avium complex from soil, water, and dusts. Microbiol Immunol 1988;32:733-739.
-4 van Ingen J, Bendien SA, de Lange WC, Hoefsloot W, Dekhuijzen PN, Boeree MJ, van Soolingen D: Clinical relevance of nontuberculous mycobacteria isolated in the Nijmegen-Arnhem region, The Netherlands. Thorax 2009;64:502-506.

5 Marras TK, Daley CL: Epidemiology of human pulmonary infection with nontuberculous mycobacteria. Clin Chest Med 2002;23: 553-567.

-6 Marras TK, Chedore P, Ying AM, Jamieson F: Isolation prevalence of pulmonary nontuberculous mycobacteria in Ontario, 1997 2003. Thorax 2007;62:661-666.

7 Koh WJ, Kwon OJ, Lee KS: Diagnosis and treatment of nontuberculous mycobacterial pulmonary diseases: a Korean perspective. J Korean Med Sci 2005;20:913-925.
8 van Ingen J, Boeree MJ, de Lange WC, Dekhuijzen PN, van Soolingen D: Impact of new American Thoracic Society diagnostic criteria on management of nontuberculous mycobacterial infection. Am J Respir Crit Care Med 2007;176:418, author reply 419.

-9 Diagnostic standards and classification of tuberculosis in adults and children. This official statement of the American Thoracic Society and the Centers for Disease Control and Prevention was adopted by the ATS Board of Directors, July 1999. This statement was endorsed by the Council of the Infectious Disease Society of America, September 1999. Am J Respir Crit Care Med 2000;161: 1376-1395.

10 Hanna BA, Ebrahimzadeh A, Elliott LB, Morgan MA, Novak SM, Rusch-Gerdes S, Acio M, Dunbar DF, Holmes TM, Rexer CH, Savthyakumar C, Vannier AM: Multicenter evaluation of the BACTEC MGIT 960 system for recovery of mycobacteria. J Clin Microbiol 1999;37:748-752. 
11 Del Portillo P, Thomas MC, Martinez E, Maranon $\mathrm{C}$, Valladares B, Patarroyo ME, Carlos Lopez M: Multiprimer PCR system for differential identification of mycobacteria in clinical samples. J Clin Microbiol 1996;34: 324-328.

12 Taylor TB, Patterson C, Hale Y, Safranek WW: Routine use of PCR-restriction fragment length polymorphism analysis for identification of mycobacteria growing in liquid media. J Clin Microbiol 1997;35:7985.

13 Management of opportunist mycobacterial infections: Joint Tuberculosis Committee guidelines 1999. Subcommittee of the Joint Tuberculosis Committee of the British Thoracic Society. Thorax 2000;55:210-218.
4 Iseman MD: Mycobacterium avium complex and the normal host: the other side of the coin. N Engl J Med 1989;321:896-898.

15 Prince DS, Peterson DD, Steiner RM, Gottlieb JE, Scott R, Israel HL, Figueroa WG, Fish JE: Infection with Mycobacterium avium complex in patients without predisposing conditions. N Engl J Med 1989;321:863-868

16 Diagnosis and treatment of disease caused by nontuberculous mycobacteria. Am Rev Respir Dis 1990;142:940-953.

17 Huang JH, Kao PN, Adi V, Ruoss SJ: Mycobacterium avium-intracellulare pulmonary infection in HIV-negative patients without preexisting lung disease: diagnostic and management limitations. Chest 1999;115: 1033-1040.

18 Erasmus JJ, McAdams HP, Farrell MA, Patz EF Jr: Pulmonary nontuberculous mycobacterial infection: radiologic manifestations. Radiographics 1999;19:1487-1505.
9 Griffith DE: Impact of new American Thoracic Society diagnostic criteria on management of nontuberculous mycobacterial infection. Am J Respir Crit Care Med 2007;176: 419.

20 Kobashi Y, Matsushima T: The microbiological and clinical effects of combined therapy according to guidelines on the treatment of pulmonary mycobacterium avium complex disease in Japan - including a follow-up study. Respiration 2007;74:394-400.

21 Fujiuchi S, Matsumoto H, Yamazaki Y, Nakao S, Takahashi M, Satoh K, Takeda A, Okamoto K, Fujita Y, Fujikane T, Shimizu T: Analysis of chest CT in patients with $\mathrm{Myco}$ bacterium avium complex pulmonary disease. Respiration 2003;70:76-81. 